\title{
Contrast Enhancement for Lipid NanoParticles (LNPs) Characterization Using Transmission Electron Microscopy (TEM)
}

Amandine Arnould ${ }^{1,2}$, Fanny Caputo ${ }^{3}$, Maria Bacia ${ }^{4}$, Isabelle Texier ${ }^{3}$, Marie Escudé ${ }^{3}$, Delphine Boutry ${ }^{3}$, Aurélien Auger ${ }^{2}$, Romain Soulas ${ }^{2}$ and Jean-François Damlencourt ${ }^{1,2}$

1. Univ. Grenoble Alpes, F-38000 Grenoble, France

2. CEA-LITEN, MINATEC Campus, F-38054 Grenoble, France

3. CEA-LETI, MINATEC Campus, F-38054 Grenoble, France

4. IBS, EPN Science Campus, F-38044 Grenoble, France

Organic nanoparticles such as Lipid NanoParticles (LNPs) are generally difficult to characterize using Transmission Electron Microscopy (TEM) due to their low contrast [1]. Indeed they are composed of very light elements (mainly carbon, hydrogen and oxygen) which weakly interact with the electron beam. Their characterization is all the more complicated since the suspension media is a liquid and is not compatible with TEM characterization (TEM column being under vacuum). To circumvent these issues, a phase-contrast enhancement device inside the microscope may be used [2]. Another alternative consists in working on sample preparation before observation [3, 4].

This study is based on the characterization by TEM of LNPs, before and after incubation with proteins, to assess their stability. The nanoparticle characterization is a key step towards their biological use, since it was demonstrated that their physico-chemical properties (size, shape, surface charge, "softness"...) completely govern their pharmacodynamics, tissue biodistribution, and metabolism [5]. The studied nanoparticles are composed of an oil (liquid) and wax (solid) lipid core stabilized in aqueous medium by surfactants (Figure 1) [6]. Particle size distribution (PSD) and shape were characterized by TEM at $200 \mathrm{kV}$ (Tecnai Osiris, FEI). In order to picture the particle morphology in their close-to-application environment, cryo-TEM and in-situ liquid TEM were performed. The in-situ liquid holder Poseidon (Protochips) that was used consists in trapping the liquid solution between two thin microchips. PSD collected by TEM methods were compared to the results obtained by batch mode DLS and after fractionation of the sample by FFF, followed by online MALS and DLS analysis.

Particles prepared using negative staining appeared to have different shape according to the oil/wax core ratio but the observation of their core/shell structure was not possible (Figure 2.a). Moreover particles tend to agglomerate during the drying step. Preparation using a cryogenic technique with or without staining to fix the dispersion state of the particle (Vitrobot, FEI) was thus used. Cryo-TEM observation showed particle distortion which may come from the blotting process before freezing because of particle softness (Figure 2.b) [7]. To avoid sample preparation artefacts, LNP were then observed thanks to the in-situ liquid holder. Contrary to liposomes which have a double membrane and are observable by insitu TEM [8], LNP could not be observed without functionalizing their surface. Particle grafting with metallic nanoparticles was thus performed on LNP surface to enhance TEM contrast. This strategy allowed the core/shell structure to be visualized at different aging step to evaluate LNP stability in different experimental conditions such as storage, injection (room) and body temperatures (Figure 2.c). Particle interaction with proteins was also performed in the in-situ holder to mimic blood protein interaction. Indeed LNP need to be stable until they have reached their site of action to deliver their active component. PSD obtained by AF4-MALS were correlated to TEM results during particle aging, confirming LNP stability. However, PSD obtained for LNP after interaction with proteins showed slight 
differences.

References:

[1] GH Michler in "Electron Microscopy of Polymers", ed. H Pasch, (Springer Laboratory, Germany) p. $175-183$

[2] R Danev and K Nagayama, Journal of Structural Biology 161 (2008), p. 211

[3] M Adrian et al, Micron 29 (1998), p. 145

[4] S Brenner and RW Horne, Biochimica et Biophysica Acta 34 (1959), p. 103

[5] N Bertrand and JC Leroux, Journal of Controlled Release 161 (2012), p. 152

[6] T Delmas et al, Journal of Colloid and Interface Science 360 (2011), p. 471

[7] D Danino, Current Opinion in Colloid an Interface Science 17 (2012), p. 316

[8] SM Hoppe et al, Langmuir 29 (2013), p. 9958

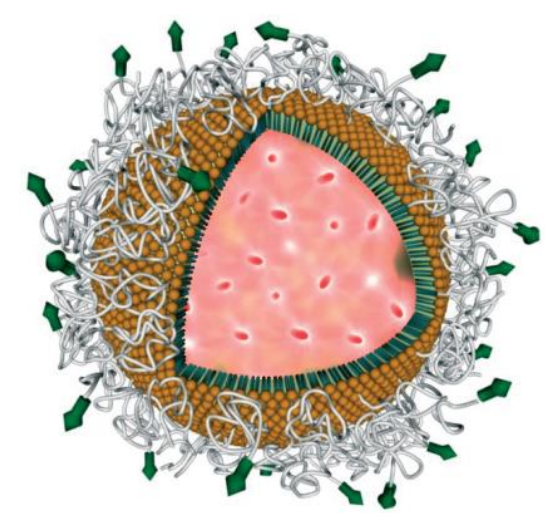

Figure 1. 3D structure of a Lipid NanoParticle.

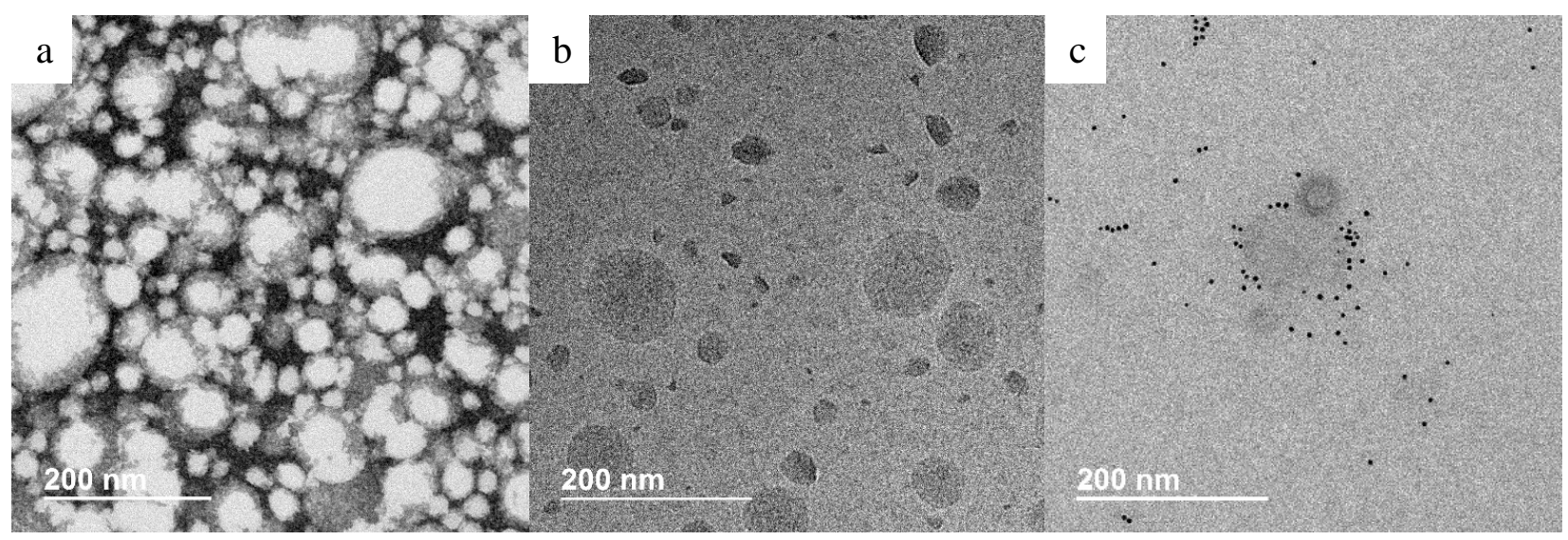

Figure 2. TEM micrographs of LNPs for different sample preparation techniques. (a) Negative staining using uranyl acetate. (b) Cryo-fixation (Vitrobot). (c) In-situ liquid holder after grafting (Poséidon, Protochips) 